\title{
Disseminated Histoplasmosis: Intestinal Multiple Ulcers without Gastrointestinal Symptoms in an Immune Competent Adult
}

\section{Linlin Zhu ${ }^{1}$, Wenyan Zhang ${ }^{2}$, Li Yang ${ }^{1}$, Tianjiao Guo ${ }^{1}$, Chang Su ${ }^{3}$ and Jinlin Yang ${ }^{1 *}$}

${ }^{1}$ Department of Gastroenterology, West China Hospital of Sichuan University, No.37 Guo Xue Xiang. Chengdu, 610041, P.R.China

${ }^{2}$ Department of Pathology, West China Hospital of Sichuan University, No.37 Guo Xue Xiang. Chengdu, 610041, P.R. China

${ }^{3}$ Department of Gastroenterology, Armed Police Forces' hospital of Chengdu, Chengdu, 610041, P.R. China

\begin{abstract}
Gastrointestinal involvement in disseminated histoplasmosis can occur at any site along the Gl tract without specific manifestations, although causes clinical symptoms in only $3-12 \%$ of patients. Intestinal histoplasmosis is the most common one, particularly at the terminal ileum as it's abundant in lymphoid tissue. Endoscopic findings in the intestines were from erythema, edema, ulcers to perforation. As the lack of specificity of these manifestations in symptoms and colonoscopy, intestinal histoplasmosis may be misdiagnosed, leading to inappropriate therapies and unnecessary surgical interventions. We report a case of immunocompetent disseminated histoplasmosis involving the colon and terminal ileum which presented fever and weight loss without gastrointestinal symptoms. So taking disseminated histoplasmosis as differential diagnosis even in immunocompetent asymptomatic patients is necessary for gastroenterologists and endoscopists.
\end{abstract}

Keywords: Disseminated histoplasmosis; Colon ulcers; Immuno competent

\section{Introduction}

Disseminated histoplasmosis (DH) is more likely to be encountered in patients whose CD4+ cell counts are $<200$ cells $/ \mathrm{mm}^{3}$, mostly found in acquired immune deficiency syndrome (AIDS). Intestinal involvement is also mostly found in immunocompromised patients. Symptoms include nausea, vomiting, abdominal pain, diarrhea and constipation. Endoscopy detected congestion, edema, ulcers, mass or perforation, being similar to intestinal tuberculosis, tumor and inflammatory bowel disease. Few physicians and endoscopists considered this disease as a differential diagnosis, so colonoscopies or fungal cultures were rarely taken. We report a healthy individual involving colon, terminal ileum and bone marrow without gastrointestinal symptoms, and Histoplasma capsulatum was finally identified.

\section{Case Report}

A 34-year-old immunocompetent male presented a medical history of intermittent fever associated with night sweats for 3 months, with the highest temperature being $40.3^{\circ} \mathrm{C}$. He complained of cough and expectoration since the first month and weight loss of $15 \mathrm{~kg}$. There were no other diseases except an occasional damp working environment.

Upon examination, his temperature was $37.7^{\circ} \mathrm{C}$, other vital signs were normal, the spleen was $5 \mathrm{~cm}$ below the left rib border and a 1 $\mathrm{cm} \times 1 \mathrm{~cm}$ lump was palpable in the left axillary fossa. Laboratory examinations revealed a hemoglobin level of $9.8 \mathrm{~g} / \mathrm{dL}$, a platelet count of $92,000 / \mathrm{mm}^{3}$, a white blood cell count of $3,200 / \mathrm{mm}^{3}$ and an ESR of $20 \mathrm{~mm} / \mathrm{hr}$. Fecal occult blood test (FOBT) was weakly positive. HBV and HCV serology was negative. Multiple human immunodeficiency virus (HIV) tests during the hospital were negative. CD4 count was 240/ $\mathrm{mm}^{3}$. Tumor markers (AFP, CEA, CA19-9, CA-125) and the chest CT were normal. Both abdominal CT scan and ultrasonography revealed hepatosplenomegaly. Repeated blood, sputum, urine, stool and bone marrow cultures were negative. Colonoscopy showed edematous mucosa, diffuse nodular changes accompanying aphthoid ulcers measuring $0.5-1.0 \mathrm{~cm}$, leading to intestinal strictures. Thus, the colonoscopy could not continue after ascending colon (Figure 1A). The second examination was carried out after 45 days, on the 44 th day of the patient's treatment with amphotericin $\mathrm{B}$. That is, we treated the patient with amphotericin B on the second day after the first colonoscopy. As a result, the second colonoscopy entered over $10 \mathrm{~cm}$ beyond the terminal
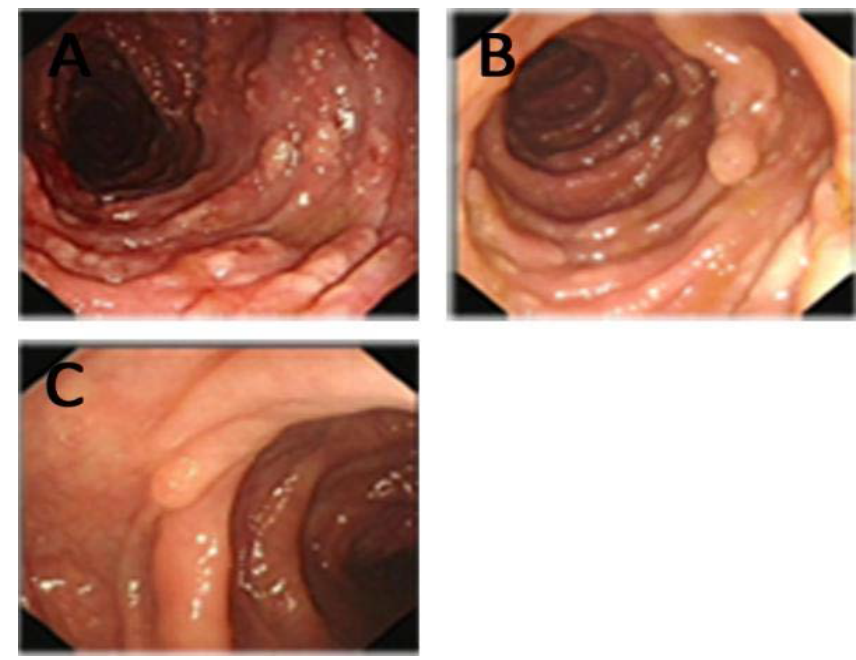

Figure 1:a Descending Colon appearance in endoscopy

A) the colon was congestion, edema, small ulcers under the first colonoscopy. B)A little relief but scattered nodular changes after being treated with amphotericin B deoxycholate.

C)Alllesions disappearedayearlater.

*Corresponding author: Jinlin Yang, Department of Gastroenterology, West China Hospital of Sichuan University, No.37 Guo Xue Xiang. Chengdu, 610041 P.R. China, Tel: 18980602058; E-mail: mouse-577@163.com

Received February 25, 2014; Accepted March 20, 2014; Published March 22 , 2014

Citation: Linlin Zhu, Wenyan Zhang, Li Yang, Tianjiao Guo, Chang Su, et al. (2014) Disseminated Histoplasmosis: Intestinal Multiple Ulcers without Gastrointestinal Symptoms in an Immune Competent Adult. J Cytol Histol 5 : 231. doi:10.4172/2157-7099.1000231

Copyright: (c) 2014 Linlin Zhu, et al. This is an open-access article distributed under the terms of the Creative Commons Attribution License, which permits unrestricted use, distribution, and reproduction in any medium, provided the original author and source are credited. 
Citation: Linlin Zhu, Wenyan Zhang, Li Yang, Tianjiao Guo, Chang Su, et al. (2014) Disseminated Histoplasmosis: Intestinal Multiple Ulcers without Gastrointestinal Symptoms in an Immune Competent Adult. J Cytol Histol 5: 231. doi:10.4172/2157-7099.1000231

ileum and the lesions were similar to that of the first colonoscopy except a little relief after being treated by amphotericin B deoxycholate (Figure 1B).

All of the biopsy specimens of the ascending colon, transverse colon, sigmoid colon, rectum, descending colon showed mucosal erosion and granuloma formation, numerous yeast-like pathogens which are positive for PAS (periodic acids chiff) and GMS (Gomori methenamine silver) but negative for Giemsa, detected in the increased number of histiocytes (Figure 2), indicating mycotic granulomatous inflammation that is consistent with histoplasmosis. Bone marrow smears showed multiple oval or round organisms with amaranth nuclei and capsule-like unstained halos around, which in context of phagocytes are highly suggestive of Histoplasmosis capsulatum (Figure 3). Bone marrow biopsy was also positive to PAS and GMS stains, which was compatible with a diagnosis of histoplasmosis. The patient was started on intravenous amphotericin $\mathrm{B}$ deoxycholate for a total of $2 \mathrm{~g}$. There was a dramatic response with a quick change in temperature within 1 week. The patient was asked to continue itraconazole for 6 months. The follow-up endoscopic and histopathological examinations were normal when he did the third times' colonoscopy a year later (Figure 1C).

\section{Discussion}

Histoplasma capsulatum is $2-4 \mu \mathrm{m}$ yeast that enters primarily into the lungs through inhalation and causes a self-limited respiratory

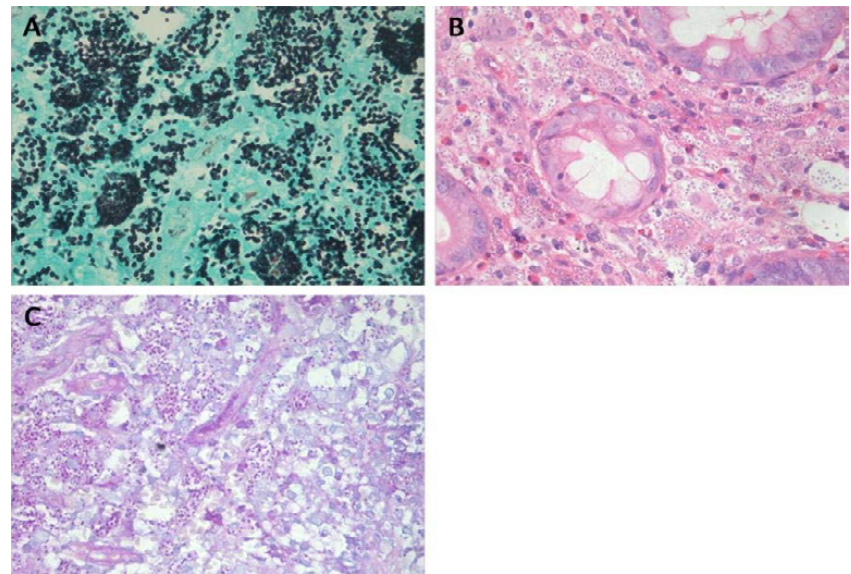

Figure 2: Numerous uniform oval-shaped yeasts suggested Histoplasma capsulatum are were found in the I amina propria stromain descending colonbiopsy.

A) Gomorime thenamine silver stain(magnification $\times 100$ )

B) HE stain(magnification $\times 40$ ).

C)PASstain (magnification $\times 40$ ).

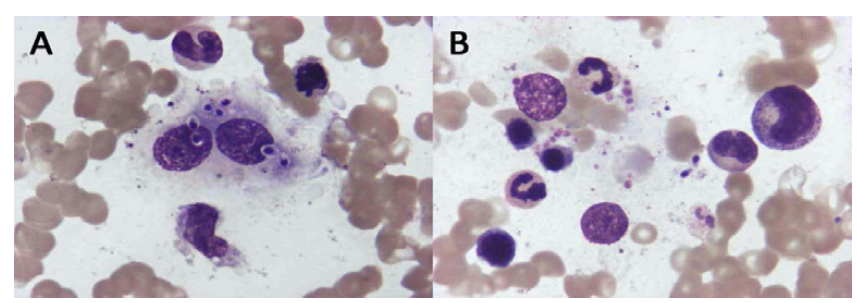

Figure 3: Wright-Giemsa stained bone marrow aspirate. There were numerous round or oval Histoplasma capsulatum of relatively uniform size in phagocyte and cytoplasm. It is round at one end and pointed at the other end. Karyon was stained fuchsia,surrounded by peri-nuclearhalos and the shape was like capsule. infection mostly in healthy individuals [1], mainly seen in endemic areas of the upper Mississippi and Ohio river valleys [2]. It is hard for macrophages to eliminate the organisms in immunodeficient patients due to absence of cellular immunity, allowing the infection to disseminate to other organs such as skinbone marrow, spleen, liver, lymphoid node, adrenal gland, renal tract, central nervous system, even the GI tract, which performs as disseminated histoplasmosis. The reported immunodeficiency conditions include AIDS [3], Hepatitis C-Infected [1], renal failure, recently used glucocorticosteriod or biological agents as infliximab and etanercept [4], but $<0.05 \%$ of patients have no obvious immunosuppressive risk factors [5], which is difficult to account for disseminated histoplasmosis. In our case, CD4 count was $240 / \mathrm{mm}^{3}$ initially but increased after the infection was treated. We considered it as an outcome of infection although we haven't found any evidence that histoplasma impaired the immune function of T-cells.

Gastrointestinal involvement occurs in $70-90 \%$ of cases of disseminated histoplasmosis who undergo an autopsy, the colon may be involved in $59.6 \%$, However, gastrointestinal histoplasmosis (GIH) is rarely recognized during life and is thought to cause clinical symptoms in only $3-12 \%$ of patients [6]. Symptoms mainly showed as abdominal pain, diarrhea, anorexia, nausea, bilious vomiting, constipation, tenesmus and abdominal tenderness [6]. Other reported GI complications include bleeding, bowel obstruction and perforation [3], even protein-losing enteropathy and hypogamma globulinaemia [7]. Doctors do not pay attention to arranging gastrointestinal endoscopy examinations due to lack of GI symptoms, leading to missed diagnoses.

Lesions seen during endoscopy, laparotomy, or autopsy include single or continuous superficial mucosal ulcerations, deep bleeding ulcers with or without frank perforation, friable and masses containing areas of necrosis, obstruction due to circumferential thickening exophytic [6]. Diffuse ulceration was detected in $85.7 \%$ (12/14) of AIDS GIH patients, $10 / 14(71.4 \%)$ being involved only the colon or cecum [8]. We all know these appearances mimic many other GI diseases as Crohn's disease, tuberculosis, carcinoma and lymphoma, it is often considered not to be histoplasmosis in the differential diagnosis leading to inappropriate therapies and unnecessary surgical interventions.

A variety of diagnostic exams including direct microscopic examination, cultures, antigen detection, serologic tests for antibodies have been described, and a tissue biopsy should be done as soon as possible. Samples can be taken from blood, bone marrow, liver, skin lesions, or any other site of infection. Cultures are positive in about $85 \%$ of cases. Multiple specimens must be cultured to achieve the highest yield [6,9]. Yielded positive culture results for blood, bronchoalveolar lavage, lymph node, liver and spleen specimens was in $52.9 \%$ of patients, but positive results from GI specimens were $90.9 \%$ (3). So specimens should be submitted for microscopic examination and fungal culture from mucosal lesions of the gastrointestinal tract identified at endoscopy besides being used to pathoscopy. GIH has excellent long term survival prospects with aggressive therapy that is anti-mycetes therapies. However, untreated disseminated histoplasmosis is almost always fatal.

In summary, it is important to arrange gastrointestinal endoscopies for $\mathrm{DH}$ cases even with a lack of gastrointestinal tract symptoms or in patients with normal immune function. Endoscopists and gastrointestinal doctors should be aware of histoplasmosis in GI tract, especially when it manifests as ulceration, and the species should be sent to culture as soon as possible. 
Citation: Linlin Zhu, Wenyan Zhang, Li Yang, Tianjiao Guo, Chang Su, et al. (2014) Disseminated Histoplasmosis: Intestinal Multiple Ulcers without Gastrointestinal Symptoms in an Immune Competent Adult. J Cytol Histol 5: 231. doi:10.4172/2157-7099.1000231

Page 3 of 3

\section{References}

1. Rodriguez-Waitkus PM, Bayat V, George E, Sule N (2013) Gastrointestinal histoplasmosis in a hepatitis C-infected individual. Mycopathologia 176: 161-164.

2. Jain S, Koirala J, Castro-Pavia F (2004) Isolated gastrointestinal histoplasmosis: case report and review of the literature. South Med J 97: 172-174.

3. Suh KN, Anekthananon T, Mariuz PR (2001) Gastrointestinalhistoplasmosis in patients with AIDS: case report and review. Clin Infect Dis 32: 483-491.

4. Seminerio JL, Loftus EV Jr, Colombel JF, Thapa P, Sandborn WJ (2013) Infliximab for Crohn's disease: the first 500 patients followed up through 2009 Dig Dis Sci 58: 797-806.

5. Rana C, Krishnani N, Kumari N (2011) Bilateral adrenal histoplasmosis in immunocompetent patients. DiagnCytopathol 39: 294-296.ahi CJ, Wheat LJ,
Allen SD, Sarosi GA (2005) Gastrointestinalhistoplasmosis. Am J Gastroenterol 100: 220-231.

6. Kok J, Chen SC, Anderson L, Berglund L, Sleiman S, et al. (2010) Proteinlosing enteropathy and hypogammaglobulinaemia as first manifestations of disseminated histoplasmosis coincident with Nocardia infection. J Med Microbiol 59: 610-613.

7. Assi M, McKinsey DS, Driks MR, O'Connor MC, Bonacini M, et al. (2006) Gastrointestinalhistoplasmosis in the acquired immunodeficiency syndrome: report of 18 cases and literature review. DiagnMicrobiol Infect Dis 55: 195-201.

8. Kauffman CA (2007) Histoplasmosis: a clinical and laboratory update ClinMicrobiol Rev 20: 115-132. 